ROSHAN LAL, SUSHEEL KUMAR ${ }^{1}$ and SUNIL HANS ${ }^{2} 3$

\title{
On the zeros of polynomials and analytic functions
}

\begin{abstract}
For a polynomial of degree $n$, we have obtained some results, which generalize and improve upon the earlier well known results (under certain conditions). A similar result is also obtained for analytic function.
\end{abstract}

1. Introduction and statement of results. The following theorem is due to Pellet ([6], [5, p. 128]).

Theorem A. Let $q(z)=a_{0}+a_{1} z+\ldots+a_{p} z^{p}+\ldots+a_{n} z^{n}, a_{p} \neq 0$, be a polynomial of degree $n$. If the polynomial

$Q_{p}(z)=\left|a_{0}\right|+\left|a_{1}\right| z+\ldots+\left|a_{p-1}\right| z^{p-1}-\left|a_{p}\right| z^{p}+\left|a_{p+1}\right| z^{p+1}+\ldots+\left|a_{n}\right| z^{n}$, has two positive zeros $r$ and $R, r<R$, then $q(z)$ has exactly $p$ zeros in the disc

$$
|z| \leq r
$$

and no zero in the annular ring

$$
r<|z|<R .
$$

The next result is due to Jayal, Labelle and Rahman [4].

\footnotetext{
${ }^{1}$ The research of the author is supported by UGC, New Delhi; F. No. 17-52/98(SA-I).

${ }^{2}$ The research of the author is supported by CSIR, New Delhi; F. No. -9/466(95)/2007EMR-I.

${ }^{3}$ Corresponding author.

2000 Mathematics Subject Classification. 30C15, 30A10.

Key words and phrases. Polynomial, analytic function, zeros.
} 
Theorem B. Let $p(z)=\sum_{v=0}^{n} a_{v} z^{v}$ be a polynomial of degree $n$ such that $a_{n} \geq a_{n-1} \geq \ldots \geq a_{1} \geq a_{0}$.

Then $p(z)$ has all its zeros in

$$
|z| \leq \frac{a_{n}-a_{0}+\left|a_{0}\right|}{\left|a_{n}\right|}
$$

Gardner and Govil [1] improved Theorem B as follows.

Theorem C. Let $p(z)=\sum_{v=0}^{n} a_{v} z^{v}$ be a polynomial of degree $n$ such that

$$
a_{n} \geq a_{n-1} \geq \ldots \geq a_{1} \geq a_{0} .
$$

Then $p(z)$ has all its zeros in the annular ring

$$
\frac{\left|a_{0}\right|}{a_{n}-a_{0}+\left|a_{n}\right|} \leq|z| \leq \frac{a_{n}-a_{0}+\left|a_{0}\right|}{\left|a_{n}\right|} .
$$

Recently Jain [3] proved the following result for the upper bound involving coefficients of the polynomial.

Theorem D. Let $q(z)=a_{0}+a_{1} z+\ldots+a_{p-1} z^{p-1}+a_{p} z^{p}+\ldots+a_{n} z^{n}$, be a polynomial of degree $n$ such that $a_{p} \neq a_{p-1}$ for some $p \in\{1,2, \ldots, n\}$. Set

$$
M=M_{p}:=\sum_{j=p+1}^{n}\left|a_{j}-a_{j-1}\right|+\left|a_{n}\right| \quad(1 \leq p \leq n-1), M_{n}:=\left|a_{n}\right|
$$

and

$$
m=m_{p}:=\sum_{j=1}^{p-1}\left|a_{j}-a_{j-1}\right| \quad(2 \leq p \leq n), m_{1}:=0 .
$$

Suppose that

$$
\frac{p}{M} \frac{\left|a_{p}-a_{p-1}\right|}{p+1}<1
$$

and that

$$
\left|a_{0}\right|+m \frac{p}{M} \frac{\left|a_{p}-a_{p-1}\right|}{p+1}<\left(\frac{p}{M}\right)^{p}\left(\frac{\left|a_{p}-a_{p-1}\right|}{p+1}\right)^{p+1} .
$$

Then $q(z)$ has at least $p$ zeros in

$$
|z|<\frac{p}{M} \frac{\left|a_{p}-a_{p-1}\right|}{p+1} .
$$

Jain [3] again, in the same paper proved the following.

Theorem E. Let $q(z)=a_{0}+a_{1} z+\ldots+a_{p-1} z^{p-1}+a_{p} z^{p}+\ldots+a_{n} z^{n}$, be a polynomial of degree $n$ such that $a_{p} \neq a_{p-1}$ for some $p \in\{1,2, \ldots, n-1\}$,

$$
\left|\arg a_{k}-\beta\right| \leq \alpha \leq \frac{\pi}{2},
$$


$k=0,1,2 \ldots, n$, for some real $\beta$ and $\alpha$ and

$$
\left|a_{n}\right| \geq\left|a_{n-1}\right| \geq \ldots \geq\left|a_{1}\right| \geq\left|a_{0}\right| .
$$

Let

$$
L=L_{p}:=\left|a_{n}\right|+\left(\left|a_{n}\right|-\left|a_{p}\right|\right) \cos \alpha+\sum_{j=p+1}^{n}\left(\left|a_{j}\right|+\left|a_{j-1}\right|\right) \sin \alpha
$$

and

$l=l_{p}:=\left(\left|\alpha_{p-1}\right|-\left|a_{0}\right|\right) \cos \alpha+\sum_{j=1}^{p-1}\left(\left|a_{j}\right|+\left|a_{j-1}\right|\right) \sin \alpha \quad(2 \leq p \leq n-1), l_{1}:=0$.

Suppose that

$$
\left|a_{0}\right|+l \frac{p}{L} \frac{\left|a_{p}-a_{p-1}\right|}{p+1}<\left(\frac{p}{L}\right)^{p}\left(\frac{\left|a_{p}-a_{p-1}\right|}{p+1}\right)^{p+1} .
$$

Then $q(z)$ has at least $p$ zeros in

$$
|z|<\frac{p}{L} \frac{\left|a_{p}-a_{p-1}\right|}{p+1}
$$

In this paper, firstly we prove the following.

Theorem 1. Let $q(z)=a_{0}+a_{1} z+a_{2} z^{2}+\ldots+a_{p-1} z^{p-1}+a_{p} z^{p}+\ldots+a_{n} z^{n}$ be a polynomial of degree $n$ such that $a_{p} \neq a_{p-1}$ for some $p \in\{1,2, \ldots, n\}$, with coefficients $a_{j}, j=0,1,2, \ldots, n$, satisfying

$$
a_{n} \geq a_{n-1} \geq \ldots \geq a_{p}>a_{p-1} \geq \ldots \geq a_{1} \geq a_{0}
$$

and

(4) $\left(\frac{p}{M_{1}}\right)^{p}\left(\frac{a_{p}-a_{p-1}}{p+1}\right)^{p+1}>\left|a_{0}\right|+\frac{p}{M_{1}}\left(\frac{a_{p}-a_{p-1}}{p+1}\right)\left(a_{p-1}-a_{0}\right)$ where $M_{1}=a_{n}+\left|a_{n}\right|-a_{p}$.

Then $q(z)$ has at least $p$ zeros in

$$
\frac{\left|a_{0}\right|}{a_{n}-a_{0}+\rho_{1}^{n}\left|a_{n}\right|} \leq|z|<\rho_{1}=\frac{p}{\left(\left|a_{n}\right|-a_{n}+a_{p}\right)} \frac{\left(a_{p}-a_{p-1}\right)}{(p+1)} .
$$

Remark 1. In Theorem 1, we have

$$
M_{1}=\left|a_{n}\right|+a_{n}-a_{p}
$$

for $1 \leq p \leq(n-1)$ and $M_{1}=\left|a_{n}\right|$ for $p=n$. The value $M_{1}=\left|a_{n}\right|+a_{n}-a_{p}$ serves the purpose for $1 \leq p \leq n$ (see also equality (1.6) of Jain [3]).

For the case $p=n$, in Theorem 1, we have the following. 
Corollary 1. Let $q(z)=a_{0}+a_{1} z+\ldots+a_{n} z^{n}$ be a polynomial of degree $n$ such that

$$
a_{n}>a_{n-1} \geq a_{n-2} \geq \ldots \geq a_{1} \geq a_{0},
$$

and

$$
\left(\frac{n}{\left|a_{n}\right|}\right)^{n}\left(\frac{a_{n}-a_{n-1}}{n+1}\right)^{n+1}>\left|a_{0}\right|+\frac{n}{n+1}\left(\frac{a_{n}-a_{n-1}}{\left|a_{n}\right|}\right)\left(a_{n-1}-a_{0}\right)
$$

then $q(z)$ has all its zeros in

$$
\frac{\left|a_{0}\right|}{a_{n}-a_{0}+\rho_{2}^{n}\left|a_{n}\right|} \leq|z|<\rho_{2}=\frac{n}{n+1} \frac{\left(a_{n}-a_{n-1}\right)}{\left|a_{n}\right|} .
$$

Remark 2. Corollary 1 is a refinement of Theorem B due to Joyal, Labelle and Rahman [4] as well as Theorem $\mathrm{C}$ due to Gardner and Govil [1] under the conditions (6) and (7).

As it can be shown easily from (8) and (2) that

$$
\frac{n}{n+1} \frac{\left(a_{n}-a_{n-1}\right)}{\left|a_{n}\right|}<\frac{a_{n}-a_{0}+\left|a_{0}\right|}{\left|a_{n}\right|}
$$

is always true.

And also

$$
\frac{\left|a_{0}\right|}{a_{n}-a_{0}+\rho_{2}^{n}\left|a_{n}\right|}>\frac{\left|a_{0}\right|}{a_{n}+\left|a_{n}\right|-a_{0}}
$$

for

$$
\rho_{2}=\frac{n}{n+1} \frac{a_{n}-a_{n-1}}{\left|a_{n}\right|} .
$$

Remark 3. If we take $a_{0}>0$, then Corollary 1 gives a refinement of a result due to Jain [3, Corollary 1].

Instead of proving Theorem 1, we prove the following result. Theorem 1 can be proved in a similar way as the next result (Theorem 2) except the only change that is in Theorem $1 p \in\{1,2, \ldots, n\}$.

Theorem 2. Let $q(z)=a_{0}+a_{1} z+\ldots+a_{p-1} z^{p-1}+a_{p} z^{p}+\ldots a_{n} z^{n}$ be a polynomial of degree $n$ such that $a_{p} \neq a_{p-1}$ for some $p \in\{1, \ldots, n-1\}$, with the coefficients $a_{j}, j=0,1, \ldots, n$, for some $K \geq 1$, satisfying

$$
K a_{n} \geq a_{n-1} \geq \ldots \geq a_{p}>a_{p-1} \geq \ldots \geq a_{1} \geq a_{0}
$$

and

$$
\left(\frac{p}{M_{2}}\right)^{p}\left(\frac{a_{p}-a_{p-1}}{p+1}\right)^{p+1}>\left|a_{0}\right|+\frac{p}{M_{2}}\left(\frac{a_{p}-a_{p-1}}{p+1}\right)\left(a_{p-1}-a_{0}\right),
$$

where

$$
M_{2}=K\left(a_{n}+\left|a_{n}\right|\right)-a_{p}
$$


Then $q(z)$ has at least $p$ zeros in

$$
\frac{\left|a_{0}\right|}{K a_{n}+(K-1)\left|a_{n}\right|-a_{0}+\rho_{3}^{n}\left|a_{n}\right|} \leq|z| \leq \rho_{3}=\frac{p}{M_{2}} \frac{\left(a_{p}-a_{p-1}\right)}{p+1}
$$

where we assume that $\rho_{3}<1$.

For the case $a_{0}>0$, we have the following.

Corollary 2. Let $q(z)=a_{0}+a_{1} z+a_{2} z^{2}+\ldots+a_{p-1} z^{p-1}+a_{p} z^{p}+\ldots+a_{n} z^{n}$ be a polynomial of degree $n$, with the condition $a_{p} \neq a_{p-1}$ and for some $p \in\{1,2, \ldots, n-1\}, K \geq 1$ satisfying

$$
K a_{n} \geq a_{n-1} \geq \ldots \geq a_{p}>a_{p-1} \geq \ldots \geq a_{0}>0
$$

and

$$
\left(\frac{p}{M_{3}}\right)^{p}\left(\frac{a_{p}-a_{p-1}}{p+1}\right)^{p+1}>a_{0}+\frac{p}{M_{3}}\left(\frac{a_{p}-a_{p-1}}{p+1}\right)\left(a_{p-1}-a_{0}\right),
$$

where

$$
M_{3}=2 K a_{n}-a_{p} .
$$

Then $q(z)$ has at least $p$ zeros in

$$
\frac{a_{0}}{\left(2 K-1+\rho_{4}^{n}\right) a_{n}-a_{0}} \leq|z|<\rho_{4}=\frac{p}{p+1} \frac{\left(a_{p}-a_{p-1}\right)}{\left(2 K a_{n}-a_{p}\right)} .
$$

Remark 4. As $\frac{a_{p}-a_{p-1}}{2 K a_{n}-a_{p}}<1$ (by (13)), we have $\frac{p}{p+1}\left(\frac{a_{p}-a_{p-1}}{2 K a_{n}-a_{p}}\right)=\rho_{4}<1$.

For the polynomials with complex coefficients, we have been able to prove the following.

Theorem 3. Let $q(z)=a_{0}+a_{1} z+\ldots+a_{p-1} z^{p-1}+a_{p} z^{p}+\ldots+a_{n} z^{n}$ be a polynomial of degree $n$ such that $a_{p} \neq a_{p-1}$ for some $p \in\{1,2, \ldots, n-1\}$, for some real $\beta$ and $\alpha$

$$
\left|\arg a_{j}-\beta\right| \leq \alpha \leq \frac{\pi}{2}, \quad j=0,1, \ldots, n,
$$

and for some $K \geq 1$,

$$
K\left|a_{n}\right| \geq\left|a_{n-1}\right| \geq \ldots \geq\left|a_{1}\right| \geq\left|a_{0}\right|
$$

and

$$
\left(\frac{p}{M_{4}}\right)^{p}\left(\frac{\left|a_{p}-a_{p-1}\right|}{p+1}\right)^{p+1}>\left|a_{0}\right|+\frac{p}{M_{4}} \frac{\left|a_{p}-a_{p-1}\right|}{(p+1)} m^{\prime}
$$

where

$$
\begin{aligned}
M_{4}= & K\left|a_{n}\right|+\left(K\left|a_{n}\right|-\left|a_{p}\right|\right) \cos \alpha+\left(K\left|a_{n}\right|+\left|a_{n-1}\right|\right) \sin \alpha \\
& +\sum_{j=p+1}^{n-1}\left(\left|a_{j}\right|+\left|a_{j-1}\right|\right) \sin \alpha,
\end{aligned}
$$




$$
m^{\prime}=\left(\left|a_{p-1}\right|-\left|a_{0}\right|\right) \cos \alpha+\sum_{j=1}^{p-1}\left(\left|a_{j}\right|+\left|a_{j-1}\right|\right) \sin \alpha .
$$

Then $q(z)$ has at least $p$ zeros in

$$
|z|<\rho_{5}=\frac{p}{p+1} \frac{\left(\left|a_{p}-a_{p-1}\right|\right)}{M_{4}} .
$$

Remark 5. In the case $K=1$, the above theorem reduces to Theorem E due to Jain [3].

Remark 6. $\rho_{5}<1$, as can be verified by using (19), (17) and Lemma 1.

Now we turn to the study of zeros of an analytic function. In this direction, we have been able to prove the following.

Theorem 4. Let the function $f(z)=\sum_{j=0}^{\infty} a_{j} z^{j}(\not \equiv 0)$ be analytic in $|z| \leq$ $\rho_{6}$, for some $p \in N$ such that $\frac{a_{p-1}}{a_{p}}<2+\frac{1}{p}$. Assume that

$$
a_{0} \geq a_{1} \geq \ldots \geq a_{p-1}>a_{p} \geq a_{p+1} \geq \ldots
$$

and

$$
\left(\frac{p}{a_{p}}\right)^{p}\left(\frac{a_{p-1}-a_{p}}{p+1}\right)^{p+1}>\left|a_{0}\right|+\frac{p}{a_{p}}\left(\frac{a_{p-1}-a_{p}}{p+1}\right)\left(a_{0}-a_{p-1}\right) .
$$

Then the function $f(z)$ has at least $p$ zeros in

$$
|z|<\rho_{6}=\frac{p}{p+1}\left(\frac{a_{p-1}-a_{p}}{a_{p}}\right) .
$$

2. Lemma. For the proof of the theorems, we need the following lemma.

Lemma 1. If $a_{j}$ is any complex number with

$$
\left|\arg a_{j}-\beta\right| \leq \alpha \leq \frac{\pi}{2},
$$

for certain real $\beta$ and $\alpha$, then

$$
\left|a_{j}-a_{j-1}\right| \leq|| a_{j}|-| a_{j-1}|| \cos \alpha+\left(\left|a_{j}\right|+\left|a_{j-1}\right|\right) \sin \alpha .
$$

This lemma is due to Govil and Rahman (proof of Theorem 2 of [2]).

\section{Proofs of Theorems.}

Proof of Theorem 2. Consider 


$$
\begin{aligned}
g(z)= & (1-z) q(z) \\
= & (1-z)\left(a_{0}+a_{1} z+\ldots+a_{p-1} z^{p-1}+a_{p} z^{p}+\ldots+a_{n} z^{n}\right) \\
= & a_{0}+\sum_{j=1}^{p-1}\left(a_{j}-a_{j-1}\right) z^{j}+\left(a_{p}-a_{p-1}\right) z^{p} \\
& +\sum_{j=p+1}^{n}\left(a_{j}-a_{j-1}\right) z^{j}-a_{n} z^{n+1} . \\
= & \phi(z)+\psi(z),
\end{aligned}
$$

where

$$
\phi(z)=a_{0}+\sum_{j=1}^{p-1}\left(a_{j}-a_{j-1}\right) z^{j}
$$

and

$$
\psi(z)=\left(a_{p}-a_{p-1}\right) z^{p}+\sum_{j=p+1}^{n}\left(a_{j}-a_{j-1}\right) z^{j}-a_{n} z^{n+1} .
$$

Now for $|z|=\rho_{3}\left(\rho_{3}<1\right.$ (as assumed)) and $p \leq n-1$,

$$
\begin{aligned}
&|\psi(z)| \geq\left|a_{p}-a_{p-1}\right| \rho_{3}^{p}-\left\{\sum_{j=p+1}^{n}\left|a_{j}-a_{j-1}\right| \rho_{3}^{j}+\left|a_{n}\right| \rho_{3}^{n+1}\right\} \\
& \geq\left(a_{p}-a_{p-1}\right) \rho_{3}^{p}-\rho_{3}^{p+1}\left\{\left|a_{n}\right| \rho_{3}^{n-p}+\left|a_{n}-a_{n-1}\right| \rho_{3}^{n-p}\right.\left.+\sum_{j=p+1}^{n-1}\left|a_{j}-a_{j-1}\right| \rho_{3}^{j-(p+1)}\right\} \\
& \geq\left(a_{p}-a_{p-1}\right) \rho_{3}^{p}-\rho_{3}^{p+1}\left\{\left|a_{n}\right|+\left|K a_{n}-a_{n-1}\right|\right.\left.+(K-1)\left|a_{n}\right|+\sum_{j=p+1}^{n-1}\left|a_{j}-a_{j-1}\right|\right\} \\
&=\left(a_{p}-a_{p-1}\right) \rho_{3}^{p}-\rho_{3}^{p+1}\left\{\left|a_{n}\right|+K a_{n}-a_{n-1}\right. \\
&\left.+(K-1)\left|a_{n}\right|+a_{n-1}-a_{p}\right\} \\
&=\left(a_{p}-a_{p-1}\right) \rho_{3}^{p}-\rho_{3}^{p+1}\left\{K\left(a_{n}+\left|a_{n}\right|\right)-a_{p}\right\} \\
&=\left(\frac{p}{M_{2}}\right)^{p}\left(\frac{a_{p}-a_{p-1}}{p+1}\right)^{p+1}
\end{aligned}
$$




$$
\begin{aligned}
& >\left|a_{0}\right|+\frac{p}{M_{2}}\left(\frac{a_{p}-a_{p-1}}{p+1}\right)\left(a_{p-1}-a_{0}\right) \text { by }(10) . \\
& =\left|a_{0}\right|+\rho_{3}\left(a_{p-1}-a_{0}\right) .
\end{aligned}
$$

Thus for $|z|=\rho_{3}$,

$$
|\psi(z)|>\left|a_{0}\right|+\rho_{3}\left(a_{p-1}-a_{0}\right) .
$$

On the other hand, for $|z|=\rho_{3}$,

$$
\begin{aligned}
|\phi(z)| & \leq\left|a_{0}\right|+\sum_{j=1}^{p-1}\left|a_{j}-a_{j-1}\right| \rho_{3}^{j} \\
& \leq\left|a_{0}\right|+\rho_{3}\left(a_{p-1}-a_{0}\right) .
\end{aligned}
$$

From (26) and (27) it follows that $|\psi(z)|>|\phi(z)|$ for $|z|=\rho_{3}$. By Rouche's theorem, $g(z)=\phi(z)+\psi(z)$ and $\psi(z)$ has same number of zeros in $|z|<\rho_{3}$. But $\psi(z)$ has at least $p$ zeros in $|z|<\rho_{3}$. Therefore $g(z)$ and hence $q(z)$ has at least $p$ zeros in

$$
|z|<\rho_{3}
$$

This proves one part of Theorem 2 .

Now it remains to prove that there are no zeros of $q(z)$ in

$$
|z|<\frac{\left|a_{0}\right|}{K a_{n}+(K-1)\left|a_{n}\right|-a_{0}+\rho_{3}^{n}\left|a_{n}\right|} .
$$

Let

$$
\begin{aligned}
g(z) & =(1-z) q(z)=a_{0}+\sum_{j=1}^{n}\left(a_{j}-a_{j-1}\right) z^{j}-a_{n} z^{n+1} \\
& =a_{0}+h(z) .
\end{aligned}
$$

Now for $|z|=\rho_{3},\left(\rho_{3}<1\right)$ we have

$$
\begin{aligned}
\max _{|z|=\rho_{3}}|h(z)| & \leq \sum_{j=1}^{n}\left|a_{j}-a_{j-1}\right| \rho_{3}^{j}+\left|a_{n}\right| \rho_{3}^{n+1} \\
& \leq \rho_{3}\left\{\left|a_{n}-a_{n-1}\right|+\sum_{j=1}^{n-1}\left|a_{j}-a_{j-1}\right|+\left|a_{n}\right| \rho_{3}^{n}\right\} \\
& \leq \rho_{3}\left\{\left|K a_{n}-a_{n-1}+a_{n}-K a_{n}\right|+a_{n-1}-a_{0}+\left|a_{n}\right| \rho_{3}^{n}\right\} \\
& \leq \rho_{3}\left\{K a_{n}+(K-1)\left|a_{n}\right|-a_{0}+\rho_{3}^{n}\left|a_{n}\right|\right\} .
\end{aligned}
$$

Since $h(0)=0, h(z)$ is analytic in $|z| \leq \rho_{3}$, by Schwarz lemma we have

$$
|h(z)| \leq\left\{K a_{n}+(K-1)\left|a_{n}\right|-a_{0}+\rho_{3}^{n}\left|a_{n}\right|\right\}|z|
$$


if $|z| \leq \rho_{3}$. Now from (28) we see that for $|z| \leq \rho_{3}$,

$$
\begin{aligned}
|g(z)| & \geq\left|a_{0}\right|-|h(z)| \\
& \geq\left|a_{0}\right|-\left\{K a_{n}+(K-1)\left|a_{n}\right|-a_{0}+\rho_{3}^{n}\left|a_{n}\right|\right\}|z| \\
& >0,
\end{aligned}
$$

if

$$
|z|<\frac{\left|a_{0}\right|}{K a_{n}+(K-1)\left|a_{n}\right|-a_{0}+\rho_{3}^{n}\left|a_{n}\right|} .
$$

This proves the theorem completely.

Proof of Theorem 3. Consider the polynomial

$$
\begin{aligned}
g(z)= & (1-z) q(z) \\
= & a_{0}+\sum_{j=1}^{p-1}\left(a_{j}-a_{j-1}\right) z^{j}+\left(a_{p}-a_{p-1}\right) z^{p} \\
& +\sum_{j=p+1}^{n}\left(a_{j}-a_{j-1}\right) z^{j}-a_{n} z^{n+1} \\
= & \phi(z)+\psi(z),
\end{aligned}
$$

where

$$
\phi(z)=a_{0}+\sum_{j=1}^{p-1}\left(a_{j}-a_{j-1}\right) z^{j}
$$

and

$$
\psi(z)=\left(a_{p}-a_{p-1}\right) z^{p}+\sum_{j=p+1}^{n}\left(a_{j}-a_{j-1}\right) z^{j}-a_{n} z^{n+1} .
$$

Now for $|z|=\rho_{5}\left(\rho_{5}<1\right)$,

$$
\begin{aligned}
&|\psi(z)| \geq\left|a_{p}-a_{p-1}\right| \rho_{5}^{p}-\rho_{5}^{p+1}\left\{\left|a_{n}\right| \rho_{5}^{n-p}+\sum_{j=p+1}^{n}\left|a_{j}-a_{j-1}\right| \rho_{5}^{j-(p+1)}\right\} \\
& \geq\left|a_{p}-a_{p-1}\right| \rho_{5}^{p}-\rho_{5}^{p+1}\left\{\left|a_{n}\right|+\sum_{j=p+1}^{n}\left|a_{j}-a_{j-1}\right|\right\} \\
&=\left|a_{p}-a_{p-1}\right| \rho_{5}^{p}-\rho_{5}^{p+1}\left\{\left|a_{n}\right|+\left|a_{n}-a_{n-1}\right|+\sum_{j=p+1}^{n-1}\left|a_{j}-a_{j-1}\right|\right\} \\
&=\left|a_{p}-a_{p-1}\right| \rho_{5}^{p}-\rho_{5}^{p+1}\left\{\left|a_{n}\right|+\left|K a_{n}-a_{n-1}+a_{n}-K a_{n}\right|\right. \\
&\left.+\sum_{j=p+1}^{n-1}\left|a_{j}-a_{j-1}\right|\right\}
\end{aligned}
$$




$$
\begin{aligned}
& \geq\left|a_{p}-a_{p-1}\right| \rho_{5}^{p}-\rho_{5}^{p+1}\left\{\left|a_{n}\right|+\left|K a_{n}-a_{n-1}\right|+(K-1)\left|a_{n}\right|\right. \\
& \left.+\sum_{j=p+1}^{n-1}\left|a_{j}-a_{j-1}\right|\right\} \\
& \geq\left|a_{p}-a_{p-1}\right| \rho_{5}^{p}-\rho_{5}^{p+1}\left\{K\left|a_{n}\right|+\left(K\left|a_{n}\right|-\left|a_{n-1}\right|\right) \cos \alpha\right. \\
& +\left(K\left|a_{n}\right|+\left|a_{n-1}\right|\right) \sin \alpha+\sum_{j=p+1}^{n-1}\left[\left(\left|a_{j}\right|-\left|a_{j-1}\right|\right) \cos \alpha\right. \\
& \left.\left.\left.+\left(\left|a_{j}\right|+\left|a_{j-1}\right|\right) \sin \alpha\right]\right\} \quad \text { (by Lemma } 1\right) \\
& =\left|a_{p}-a_{p-1}\right| \rho_{5}^{p}-\rho_{5}^{p+1}\left\{K\left|a_{n}\right|+\left(K\left|a_{n}\right|-\left|a_{p}\right|\right) \cos \alpha\right. \\
& \left.+\left(K\left|a_{n}\right|+\left|a_{n-1}\right|\right) \sin \alpha+\sum_{j=p+1}^{n-1}\left(\left|a_{j}\right|+\left|a_{j-1}\right|\right) \sin \alpha\right\} \\
& =\left|a_{p}-a_{p-1}\right| \rho_{5}^{p}-\rho_{5}^{p+1} M_{4} \\
& \left.=\left(\frac{p}{M_{4}}\right)^{p}\left(\frac{\left|a_{p}-a_{p-1}\right|}{p+1}\right)^{p+1}, \quad \text { (by definition of } M_{4}\right) \text {. }
\end{aligned}
$$

Thus on $|z|=\rho_{5}$,

$$
\begin{aligned}
|\psi(z)| & \geq\left(\frac{p}{M_{4}}\right)^{p}\left(\frac{\left|a_{p}-a_{p-1}\right|}{p+1}\right)^{p+1} \\
& >\left|a_{0}\right|+\frac{p}{M_{4}}\left(\frac{\left|a_{p}-a_{p-1}\right|}{p+1}\right) m^{\prime} \\
& =\left|a_{0}\right|+\rho_{5} m^{\prime} \quad(\text { by }(18)) .
\end{aligned}
$$

Now for $|z|=\rho_{5}$,

$$
\begin{aligned}
|\phi(z)| & \leq\left|a_{0}\right|+\sum_{j=1}^{p-1}\left|a_{j}-a_{j-1}\right| \rho_{5}^{j} \leq\left|a_{0}\right|+\rho_{5} \sum_{j=1}^{p-1}\left|a_{j}-a_{j-1}\right| \\
& \leq\left|a_{0}\right|+\rho_{5}\left\{\sum_{j=1}^{p-1}\left(\left|a_{j}\right|-\left|a_{j-1}\right|\right) \cos \alpha+\sum_{j=1}^{p-1}\left(\left|a_{j}\right|+\left|a_{j-1}\right|\right) \sin \alpha\right\} \\
& =\left|a_{0}\right|+\rho_{5}\left\{\left(\left|a_{p-1}\right|-\left|a_{0}\right|\right) \cos \alpha+\sum_{j=1}^{p-1}\left(\left|a_{j}\right|+\left|a_{j-1}\right|\right) \sin \alpha\right\} \\
& =\left|a_{0}\right|+\rho_{5} m^{\prime} \quad(\text { by (20)). }
\end{aligned}
$$


Thus for $|z|=\rho_{5}$,

$$
|\phi(z)| \leq\left|a_{0}\right|+\rho_{5} m^{\prime} .
$$

From (30) and (31), we see that on $|z|=\rho_{5},|\phi(z)|<|\psi(z)|$, thereby implying by Rouche's theorem that $g(z)=\phi(z)+\psi(z)$ and $\psi(z)$ have the same number of zeros in $|z|<\rho_{5}$. Since $\psi(z)$ has at least $p$ zeros in $|z|<\rho_{5}$, this implies that $g(z)$ and hence $q(z)$ has at least $p$ zeros in $|z|<\rho_{5}=$ $\frac{p}{p+1} \frac{\left|a_{p}-a_{p-1}\right|}{M_{4}}$.

Thus the proof of Theorem 3 is completed.

Proof of Theorem 4. It is clear that $\lim _{j \rightarrow \infty} a_{j}=0$. Consider

$$
\begin{aligned}
F(z) & =(z-1) f(z) \\
& =-a_{0}+\left(a_{0}-a_{1}\right) z+\left(a_{1}-a_{2}\right) z^{2}+\ldots+\left(a_{p-1}-a_{p}\right) z^{p}+\ldots \\
& =\phi(z)+\psi(z),
\end{aligned}
$$

where

$$
\phi(z)=-a_{0}+\sum_{j=1}^{p-1}\left(a_{j-1}-a_{j}\right) z^{j}
$$

and

$$
\psi(z)=\left(a_{p-1}-a_{p}\right) z^{p}+\sum_{j=p+1}^{\infty}\left(a_{j-1}-a_{j}\right) z^{j}
$$

Now for $|z|=\rho_{6}\left(\rho_{6}<1\right.$, by hypothesis for $\left.\frac{a_{p-1}}{a_{p}}<2+\frac{1}{p}\right)$,

$$
\begin{aligned}
|\psi(z)| & \geq\left|a_{p-1}-a_{p}\right| \rho_{6}^{p}-\rho_{6}^{p+1}\left\{\sum_{j=p+1}^{\infty}\left|a_{j-1}-a_{j}\right| \rho_{6}^{j-(p+1)}\right\} \\
& \geq\left(a_{p-1}-a_{p}\right) \rho_{6}^{p}-\rho_{6}^{p+1}\left\{\sum_{j=p+1}^{\infty}\left|a_{j-1}-a_{j}\right|\right\} \\
& =\left(a_{p-1}-a_{p}\right) \rho_{6}^{p}-\rho_{6}^{p+1} a_{p} \\
& =\left(\frac{p}{a_{p}}\right)^{p}\left(\frac{a_{p-1}-a_{p}}{p+1}\right)^{p+1} \\
& >\left|a_{0}\right|+\rho_{6}\left(a_{0}-a_{p-1}\right) \quad(\text { by }(23)) .
\end{aligned}
$$

Thus for $|z|=\rho_{6}$,

$$
|\psi(z)|>\left|a_{0}\right|+\rho_{6}\left(a_{0}-a_{p-1}\right) .
$$


Now for $|z|=\rho_{6}\left(\rho_{6}<1\right)$

$$
\begin{aligned}
|\phi(z)| & \leq\left|a_{0}\right|+\sum_{j=1}^{p-1}\left|a_{j-1}-a_{j}\right| \rho_{6}^{j} \\
& \leq\left|a_{0}\right|+\rho_{6} \sum_{j=1}^{p-1}\left|a_{j-1}-a_{j}\right| \\
& =\left|a_{0}\right|+\rho_{6}\left(a_{0}-a_{p-1}\right) .
\end{aligned}
$$

Now the remaining proof of the Theorem 4 follows on the same lines of Theorem 3.

Acknowledgment. The authors are grateful to the referee for valuable suggestions.

\section{REFERENCES}

[1] Gardner, R. B., Govil, N. K., Some generalizations of the Eneström-Kakeya theorem, Acta Math. Hungar. 74 (1-2) (1997), 125-134.

[2] Govil, N. K., Rahman, Q. I., On the Eneström-Kakeya theorem, Tôhoku Math. J. 20 (1968), 126-136.

[3] Jain, V. K., On the zeros of a polynomial, Proc. Indian Acad. Sci. Math. Sci. 119 (1) (2009), 37-43.

[4] Joyal, A., Labelle, G. and Rahman, Q. I., On the location of zeros of polynomials, Canadian Math. Bull. 10 (1967), 55-63.

[5] Marden, M., The Geometry of Polynomials, Math. Surveys No. 3, Amer. Math. Soc., Providence, RI, 1966.

[6] Pellet, M.A., Sur un mode de séparation des racines des équations et la formule de Lagrange, Bull. Sci. Math. 5 (1881), 393-395.

Roshan Lal

Department of Mathematics

Government Degree College

Chaubattakhal (Pauri)

Uttrakhand 246162

India

e-mail: rlkeshtwal@yahoo.co.in

\author{
Susheel Kumar \\ Department of Mathematics \\ Faculty of Natural Sciences \\ Jamia Millia Islamia (Central University) \\ New Delhi - 110025 \\ India \\ e-mail: ahlawat_skumar@yahoo.co.in
}

Sunil Hans

Department of Mathematics

Faculty of Natural Sciences

Jamia Millia Islamia (Central University)

New Delhi - 110025

India

e-mail: sunil.hans82@yahoo.com

Received February 26, 2010 